\title{
AN IMPROVED PATTERN BASED LDA TOPIC MODELING FOR BUSINESS INTELLIGENCE
}

\author{
K Prashant Gokul ${ }^{1}$., M.Sunderrajan ${ }^{2}$ \\ ${ }^{I}$ Research Scholar, Dept. of ECE.,BIHER. \\ ${ }^{2}$ Pro-VC.,BIHER \\ ${ }^{1}$ kprashantgokul@gmail.com ,2 ${ }^{2}$ msrajan69@gmail.com,.
}

\begin{abstract}
Topic models give a helpful strategy to dimensionality decrease and exploratory data analysis in huge text corpora. Most ways to deal with topic model learning have been founded on a greatest likelihood objective. Proficient algorithms exist that endeavor to inexact this target, yet they have no provable certifications. As of late, algorithms have been presented that give provable limits, however these algorithms are not down to earth since they are wasteful and not hearty to infringement of model presumptions. In this work, we propose to consolidate the statistical topic modeling with pattern mining strategies to produce pattern-based topic models to upgrade the semantic portrayals of the conventional word based topic models. Using the proposed pattern-based topic model, clients' inclinations can be modeled with different topics and every one of which is addressed with semantically rich patterns. A tale information filtering model is proposed here. In information filtering model client information needs are made in terms of different topics where every topic is addressed by patterns. The calculation produces results similar to the best executions while running significant degrees quicker.
\end{abstract}

Keywords: Topic Model, User Interest Modeling, Pattern Mining, Information Filtering

\section{Introduction}

As of late, we have seen an increment in the amounts of accessible advanced textual data, producing new bits of knowledge and accordingly opening up promising circumstances for research along new channels. In this quickly advancing field of huge data scientific strategies, text mining has acquired critical consideration across an expansive scope of utilizations. In both scholarly community and industry, there has been a move towards research undertakings and more mind boggling research addresses that command more than the basic retrieval of data. Because of the expanding significance of artificial intelligence and its usage on advanced stages, the use of equal processing, profound learning, and pattern recognition to textual information is pivotal. A wide range of business models, statistical surveying, promoting plans, political missions, or vital dynamic are confronting an expanding need for text mining methods to address the opposition. A lot of textual data could be gathered as a piece of a research, like logical writing, records in the advertising and financial areas, talks in the field of political talk, like official missions and introduction addresses, and meeting records. Besides, online sources, like emails, web pages, web journals/miniature sites, social media posts, and remarks, give a rich wellspring of textual data for research [1].

A lot of data are likewise being gathered in semi-organized structure, for example, log records containing information from workers and organizations. Accordingly, text mining analysis is valuable for both unstructured and semi-organized textual data [6]. Data mining and text mining contrast on the kind of data they handle. While data mining handles organized data coming from frameworks, like databases, bookkeeping pages, ERP, CRM, and accounting applications, text mining manages unstructured data found in documents, emails, social media, and the web. Subsequently, the contrast between ordinary data mining and text mining is that in text mining the patterns are removed from regular language text instead of from organized databases of realities [12]. Since all the composed or spoken information can be addressed in textual structure, data mining requires a wide range of text mining instruments with regards to the translation and analysis of sentences, words, phrases, talks, cases, adverts, and explanations. This paper leads a broad analysis of text mining applications in large data investigation as utilized in different business fields and scholastic examinations. While by far most of the writing manages the streamlining of a particular text mining procedure, this paper looks to sum up the highlights of all text mining techniques, along these lines summing up the cutting edge practices and approaches on the whole the potential fields of use. It is revolved around seven key utilizations of text mining in records and addresses, meeting records, and scholarly diary articles, just as websites, emails, web journals, and social media organizing destinations; for every one of these, we, separately, give a portrayal of the field, their usefulness, the most normally utilized strategies, the 
related issues, and the related and pertinent references. The two algorithms run in polynomial time, however the limits that have been demonstrated on their example intricacy are feeble and their exact runtime execution is moderate. It is likewise muddled how they perform if the data doesn't fulfill the modeling suppositions.

\section{Related works}

Topic models are frequently used to portray plain text documents and to extricate topical substance from them. One such model, LSI, can amass words and expressions that display synonymy, e.g., vehicle and auto. The LSI strategy normally performs grid factorization over a term-document network (TF-IDF framework), 8 which addresses the event of words in documents utilizing the ideas of eigenvalue disintegration and distinguishes patterns in the connections between the document terms and ideas or topics. Nonetheless, we utilized LSI to group inquiries under a given overview topic and to fabricate topical inquiry banks on the grounds that probabilistic topic models, for example, LDA and HDP are less powerful in modeling little documents [5].

In the probabilistic topic modeling setting, a topic is addressed by a multinomial appropriation of words in a jargon. Topic modeling permits us to address the properties of an enormous collection of documents containing various words with a little collection of topics. Each document is portrayed by a mixture of topics, and words are looked over the multinomial that outcomes from the mixture of that document's topic multinomials. Topic models are intended to deal with both polysemy and synonymy. We use topic modeling algorithms, for example, HDP [3] and LDA [2] to find topics from studies. Overview questions are generally short, which varies generously from ordinary document information retrieval and mining issues. In [8] tried the materialness of topic-modelingbased ways to deal with a Twitter dataset, and found that the limited lengths of tweets keeps them from abusing their maximum capacity. Conglomerating tweets to prepare the topic model can yield an improved arrangement of topics. The research work of in [6] examines comparative perceptions on an alternate Twitter dataset. In this paper, we utilized a comparative technique to model overviews. We total the inquiries of each review and believe that to be a solitary document for topic modeling.

In [4], presented and reviewed the field of assessment mining and notion analysis, which encourages us to notice a components from the scaring unstructured text. The creators examined the most broadly contemplated subject of subjectivity classification and slant which indicates whether a document is obstinate. Additionally, they portrayed viewpoint based conclusion analysis which abuses the full force of the theoretical model and examined about perspective extraction dependent on topic modeling draws near. In [5], they examined difficulties of text mining methods in information frameworks research and indigested the pragmatic utilization of topic modeling in blend with illustrative regression analysis, utilizing on the web customer surveys as a commendable data source.

Topic models have numerous applications in normal processing dialects. Numerous articles have been distributed dependent on topic modeling approaches in different subject like Social Network, software designing, Linguistic science and so forth There are a few works that have zeroed in on overview in Topic modeling. In [2], the creators introduced a review on topic modeling in software designing field to indicate how topic models have up to this point been applied to at least one software repositories. They zeroed in on articles composed between Dec 1999 to Dec 2014 and overviewed 167 article that utilizing topic modeling in software designing region. They recognized and exhibit the research patterns in mining unstructured repositories by topic models. They found that the greater part of studies zeroed in on just a set number of software designing errand and furthermore most examinations utilize just essential topic models. In [3], the creators zeroed in on overview in Topic Models with delicate clustering capacities in text corpora and explored essential ideas and existing models classification in different classes with boundary assessment and execution assessment measures. Moreover, the creators introduced a few uses of topic models for modeling text corpora and talked about a few open issues and future bearings.

Topic models are frequently used to portray plain text documents and to extricate topical substance from them. One such model, LSI, can amass words and expressions that display synonymy, e.g., vehicle and auto. The LSI strategy normally performs grid factorization over a term-document network (TF-IDF framework), 8 which addresses the event of words in documents utilizing the ideas of eigenvalue disintegration and distinguishes patterns in the connections between the document terms and ideas or topics. Nonetheless, we utilized LSI to group inquiries under a given overview topic and to fabricate topical inquiry banks on the grounds that probabilistic topic models, for example, LDA and HDP are less powerful in modeling little documents [5].

In the probabilistic topic modeling setting, a topic is addressed by a multinomial appropriation of words in a jargon. Topic modeling permits us to address the properties of an enormous collection of documents containing various words with a little collection of topics. Each document is portrayed by a mixture of topics, and words are looked over the multinomial that outcomes from the mixture of that document's topic multinomials. Topic models are intended to deal with both polysemy and synonymy. We use topic modeling algorithms, for example, HDP [3] and LDA [2] to find topics from studies. Overview questions are generally short, which varies generously from ordinary document information retrieval and mining issues. In [8] tried the materialness of topic-modeling- 
based ways to deal with a Twitter dataset, and found that the limited lengths of tweets keeps them from abusing their maximum capacity. Conglomerating tweets to prepare the topic model can yield an improved arrangement of topics. The research work of in [6] examines comparative perceptions on an alternate Twitter dataset. In this paper, we utilized a comparative technique to model overviews. We total the inquiries of each review and believe that to be a solitary document for topic modeling.

In [13] portrayed a few strategies to perform text-mining including reviews. They clarified techniques like TF-IDF, kimplies, and various leveled clustering on the study question and answer words which depend on the R bundle tm. Here, be that as it may, we have an extensive arrangement of trials on multilingual review datasets to which we apply progressed statistical models like LDA, HDP, and LSI.

In [4], presented and reviewed the field of assessment mining and notion analysis, which encourages us to notice a components from the scaring unstructured text. The creators examined the most broadly contemplated subject of subjectivity classification and slant which indicates whether a document is obstinate. Additionally, they portrayed viewpoint based conclusion analysis which abuses the full force of the theoretical model and examined about perspective extraction dependent on topic modeling draws near. In [5], they examined difficulties of text mining methods in information frameworks research and indigested the pragmatic utilization of topic modeling in blend with illustrative regression analysis, utilizing on the web customer surveys as a commendable data source.

In [12] proposed a theory that software concerns are comparable to the latent topics found by statistical topic models. Further, they recommended that angles are those latent topics that have a high dissipating metric. The creators applied their procedure to an enormous arrangement of opensource frameworks to recognize the worldwide arrangement of topics, just as play out a more nitty gritty analysis of a couple of explicit frameworks. The creators found that latent topics with high dissipating metrics are in fact those that are ordinarily named angles in the AOP people group. In [6] presented a topic visualization apparatus, called TopicXP , which upholds intuitive investigation of found topics situated in source code. Our research contrast with different works is that, we had a profound report on topic modeling approaches dependent on LDA with the inclusion of different perspectives like applications, devices, dataset and models.

\section{Pattern Based Lda Topic Model (PLDA)}

Topic models were initially evolved as methods for consequently ordering, looking, clustering, and organizing. The little preparing set and document lengths make this a troublesome classification issue. To start, we built a fundamental Nave Bayes classifier that appoints each document D, a probability of having a place with every classification $\mathrm{cj}$, characterized as

$P(c j \mid D)=P(c j) Y$ fieFD $(P(f i \mid c j))(1)$

where FD is the list of capabilities of document $D$. The element space comprises of the two words from the text, titles and modified works of documents just as the bigrams from these strings. Bigrams are helpful here - for instance, the word "dialects" isn't so useful, however the expressions "dialects in" and "dialects of" demonstrate conversation of dialects in a specific district or family, and accordingly should lean toward the language documentation and typology classifications. Since certain classifications are essentially under-marked, rather than characterizing $\mathrm{P}(\mathrm{cj})$ as the noticed probability, we accept that the classes have a uniform circulation. The probability of a component given a class is assessed utilizing Laplace smoothing [10]:

$\mathrm{P}(\mathrm{fi} \mid \mathrm{cj})=\mathrm{ni}+1 \mathrm{n}+|\mathrm{F}|(2)$

where ni is the quantity of models named cj that have fi as a functioning component, $\mathrm{n}$ is the quantity of interesting dynamic highlights among all models named $\mathrm{cj}$, and $\mathrm{F}$ is the list of capabilities. We need to permit a paper to be put into various classes, or none, in the event that it doesn't coordinate any classification. For a particularly any-of classification task, one would regularly make a parallel classifier for each class and determine participation in each class independently [8]. We don't do this here in light of the fact that papers were marked with some however not the entirety of the classifications they may have a place with, so we can't expect that the shortfall of a name suggests that a document can be utilized as a negative model for participation to a class. All things being equal, we name a paper with some subset of classifications wherein $P(c j \mid D)$ is fundamentally more prominent than the others. To do this, we initially perform zscore standardization on the probabilities [5].

This presentation is quite more regrettable than the semisupervised Naive Bayes classifier. Nonetheless, it had the option to effectively order papers that Naive Bayes proved unable. We at that point made an extra stride of semi-oversight and removed the top consequences of our joined Naive Bayes with Wikipedia classifier and added them to the preparation set. We re-ran our unique semi-supervised Naive Bayes classifier with this new preparing set to get our end-product.

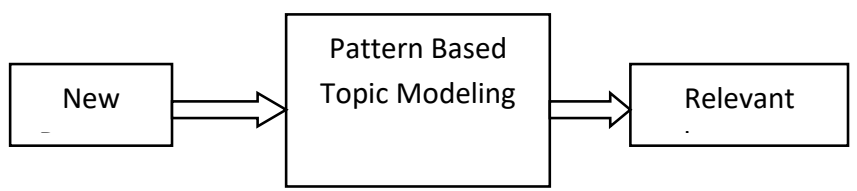

Fig. 3.1 Pattern Based Topic Modeling

Most extreme Matched Pattern-based Topic Model [8] comprises of topic disseminations portraying topic inclinations of each document or the document collection and patternbased topic portrayals introducing the semantic importance of every topic. There are mostly two stages in this model. Initial 
one is Document preparing stage and second one is Document filtering stage. During the document preparing stage client interest modeling is finished. Four stages are proposed to produce the Topic based client interest model. First Topic modeling calculation named LDA applying to each documents. LDA consequently groups documents into number of topics and every topics contain number of words dependent on their probability. Next build another value-based dataset from the consequence of LDA, which eliminates copy words. The resultant value-based dataset is the contribution to the pattern mining calculation. Next mine successive patterns by utilizing productive pattern mining calculation. Pattern conveys more information than single words. In the filtering stage, approaching documents are goes through topic modeling; pattern mining lastly the PLDA chooses greatest coordinated patterns, rather than utilizing every single found pattern. At that point contrast the approaching document pattern and preparing documents pattern. From that we can discover Maximum coordinated patterns and which is utilized for assessing significance of approaching documents. Figure 3.1 address the general design of proposed framework.

A pattern is normally characterized as a bunch of related terms or words. The patterns convey more semantic significance and are more justifiable than singular words. The possibility of the pattern-based portrayals begins from the knowledge of continuous pattern mining. It assumes a fundamental part in numerous data mining assignments coordinated toward finding fascinating patterns with regards to datasets. We accept that pattern-based portrayals are more significant and more precisely address topics than word-based portrayals. Additionally, pattern-based portrayals contain underlying information which can uncover the relationship between words. To find semantically significant patterns to address topics and documents, two stages are proposed: first and foremost, develop another value-based dataset from the LDA aftereffects of the document collection D; besides, create pattern-based portrayals from the conditional dataset to address client needs of the collection D.

\section{Algorithm 1: Two-stage pattern}

Information: a collection of documents $\mathrm{D}$; least help $\sigma \mathrm{j}$ as edge for topic $\mathrm{Zj}$; number of topics $\mathrm{V}$

Yield: pattern-based topic portrayal $X Z \mathrm{j}, \mathrm{j}=1, \bullet, \mathrm{V}$

1: Generate topic portrayal $\Phi$ and $\mathrm{Rdi}, \mathrm{Zj}$ by applying LDA to $\mathrm{D}$

2: for every topic $\mathrm{Zj} \in[\mathrm{Z1}, \mathrm{ZV}]$ do

3: Construct topical document exchange Ii $\mathrm{j}=\mathrm{n} w \mid \mathrm{w} \in \mathrm{Rdi}, \mathrm{Zj}$

o which contains the words in document di and allocated to topic $\mathrm{Zj}$

4: Construct conditional dataset $\Gamma \mathrm{j}$ for topic $\mathrm{Zj}, \Gamma \mathrm{j}=\mathrm{n} \mathrm{I} 1 \mathrm{j}, \bullet$, $\mathrm{IM}$ jo, where $\mathrm{M}$ is the quantity of unique documents in $\mathrm{D}$
5: Generate topic portrayals $\mathrm{XZj}$ for topic $\mathrm{Zj}$ utilizing a pattern mining procedure so that for each pattern $X$ in $X Z j, \operatorname{supp}(X)>$ $\sigma \mathrm{j}$

6: end for

\section{Experimental Setup}

We utilize a solitary rundown of basic English stop-words for all datasets. PLDA works on bag-of-words text portrayals, as was applied to the crude frequency esteems. In our investigations, we think about two distinctive topic modeling draws near:

1. Standard LDA, which is statistical probability model that produces document-topic and word-topic conveyance with the utilization of Dirichlet priors like alpha and beta. These priors are hyper-boundaries which are utilized to appraise document-topics thickness and topic-word thickness individually [14].

2. Dirichlet Multinomial Mixture Model (DMM), an evidently less predominant topic model, expect that a corpus can simply have a solitary topic, which is by all accounts all the more logically fitting speculation for short substance [17]. Gauge topic models use Gibbs sampling technique to deduce the topics. It is one of the sampling contingent algorithms which utilizes Markov Chain Monte Carlo (MCMC). Gibbs sampler works by disseminations of factors, back circulation as its objective appropriation to recognize the topic in the documents [15]

Since DMM and LDA has no earlier boundaries like alpha and beta to model word appropriations in corpus, it additionally results in over fitting when number of documents is expanded straightly

\section{Result}

In this part we thoroughly survey the issue of insecurity in topic modeling for standard PLDA approaches on an assorted collection of corpora, and analyze the degree to which unrivaled instatement and troupe strategies can improve the dependability of PLDA based methodologies, while likewise yielding precise models. For our examinations, we utilize a different arrangement of ten corpora, including both top notch long texts and client created content. These corpora have human annotated "groundtruth" topical classifications, permitting us to assess model accuracy. For this analysis, we used ongoing articles from business and innovation segments at New York Times. We utilized the collection of these articles as my corpus for the topic modeling exercise. Hence, each article is a document, with an obscure topic structure.

Topic coherence metrics score a solitary topic by estimating the level of semantic likeness between high probability terms in the topic. These metrics are utilized to recognize semantically interpretable topics and topics that are selfassertive antiquities of statistical induction, where the first expressed alternative ordinarily is the most pursued [16]. The 
metrics $\mathrm{Cv}$ and UMass, are two metrics that have been appeared to coordinate well with human decisions of topic quality. The two measures depend on a similar undeniable level thought, to figure the coherence of a topic as the amount of pair insightful scores over the arrangement of topic terms. These scores can be deciphered as how well the terms uphold each other as per their likeness in regard to any remaining terms. The median or the normal of the individual topic coherence is typically determined to quantify the topic coherence of the full model.

The PLD, DMM and LDA algorithms results are introduced in Figure 4.1, Figure 4.2 and Figure 4.3. A reasonable declining pattern was noticed for each of the four learned PLDA models as the quantity of topics $\mathrm{K}$ expanded. The $\mathrm{Cv}$ pattern was not monotonic in its decline notwithstanding and had nearby variety spikes for little scopes of $\mathrm{K}$, demonstrating that there can be a neighborhood ideal of various topics to finds in little neighborhood ranges of $\mathrm{K}$. The model enhanced for the performed prominently more regrettable in coherence metrics contrasted with its friends. It particularly beat the others in unmistakably doling out a solitary topic to an article on normal as demonstrated by the RS metric. The PLDA model accomplished insignificantly higher coherence scores yet performed possibly more awful in the RS metrics.

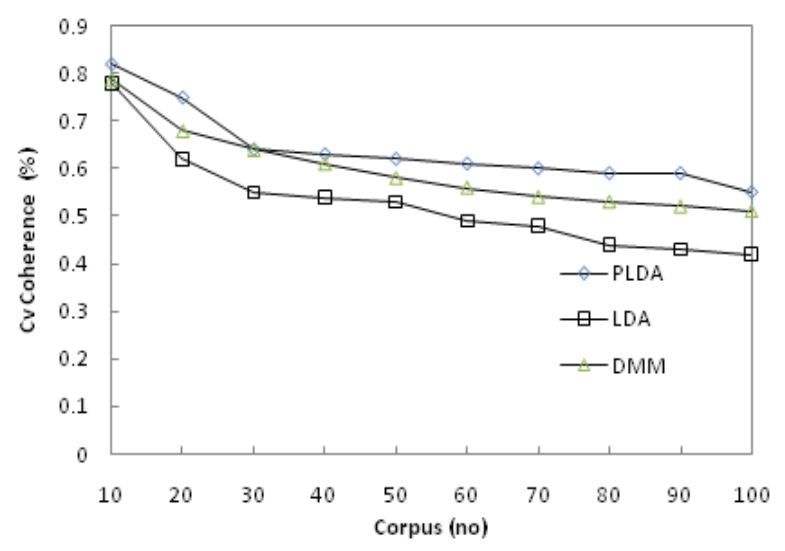

Fig 5.1 Cv Coherence

When all is said in done, the PLDA algorithms performed better or if nothing else as great as the LDA and DMM algorithms by and large, particularly on less topics. The NMF algorithms all had clear declining patterns in coherence score as the quantity of topics $\mathrm{K}$ expanded. This declining pattern was additionally noticed for LDA, particularly in UMass and RS, however it was not as articulated concerning PLDA. When looking at the PLDA and DMM, LDA model it was significantly more clear, contrasted with the overall case, that the ideal LDA and DMM didn't have this striking declining pattern. The PLDA accomplished stable coherence scores for quite a few topics up to 100 however had marginally diminishing coherence scores from 10 to 100 topics.

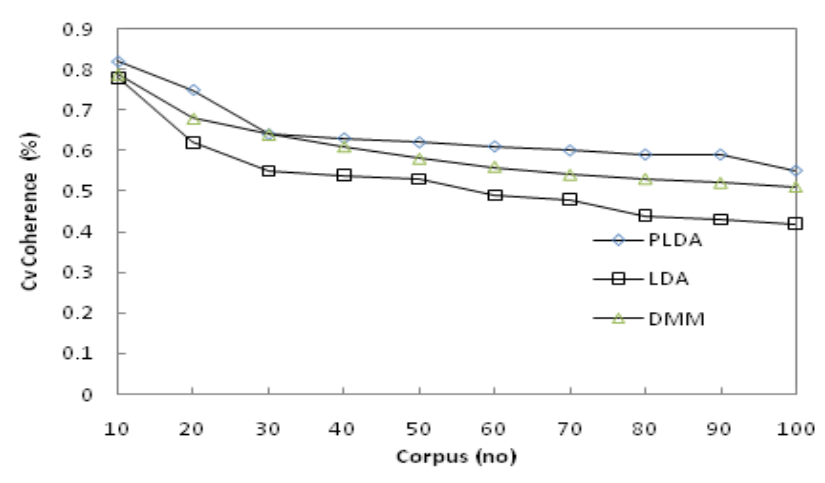

Fig .5.2 Umass Coherence

Albeit the PLDA model was better in the $\mathrm{Cv}$ coherence score than the LDA and DMM model for the lower end of the topic range, the two of them had around a similar coherence score for the higher finish of the range. For UMass these two forms were similar. The PLDA improved in the last $10 \%$ total for both coherence scores, be that as it may, and specifically for UMass contrasted with the DMM and LDA. For the RS score, the PLDA surpassed the ideal LDA by and large, while the last 10 percentile was similar. This outcome clarified that the ideal NMF model commonly performed better or as great as the ideal LDA model on normal for all scores, however performed on par or more terrible in coherence for its most confused topics. PLDA was decided to be preferable however less steady over LDA in these metrics. The RS metric likewise showed a slight bit of leeway for PLDA for allotting articles to topic

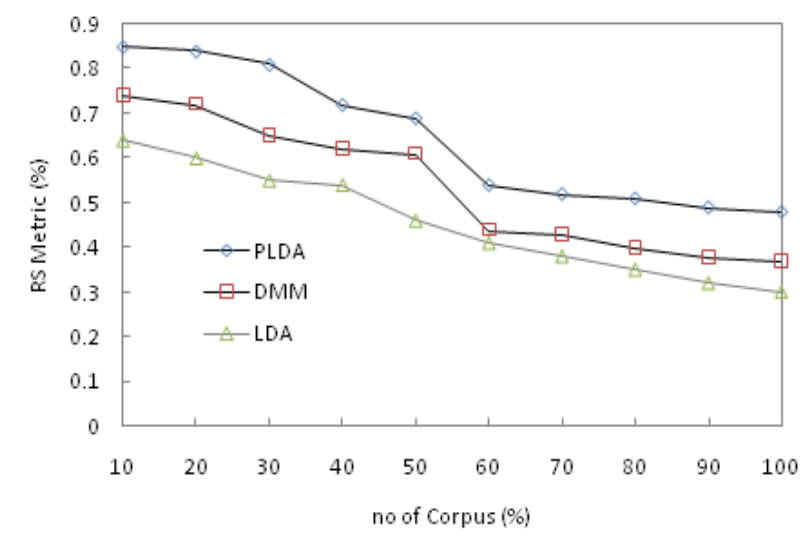

Fig .5.3 RS Metric

\section{Conclusion}

Topic modeling is quite possibly the most mainstream probabilistic text modeling strategies. The topics that are found during topic modeling are addressed by the dispersion of words. Its significance is immediately acknowledged by machine learning and text mining networks. An essential presumption for these methodologies is that the documents in the collection are about single topic. In any case, truly this isn't really the situation. Patterns can convey more semantic 
significance than single words. Here topic modeling in the field of information filtering is fundamentally thought of. The Pattern based LDA (PLDA) Topic Modeling can think about different topics inside a document. The patterns in the PLDA are very much organized so the most extreme coordinated patterns can be effectively chosen and used to address and rank documents.

\section{References}

[1] Surveymonkey, “ Available: http://www. surveymonkey.com”, 2012

[2] Blei, Jordan, "Latent Dirichlet allocation," JMLR, vol. 3, pp. 993-1022, 2003.

[3] Teh, Jordan, and Blei, "Hierarchical Dirichlet processes," Journal of the American Statistical Association, vol. 101, no. 476, pp. 1566-1581, 2006.

[4] Bezdek, "Pattern Recognition with Fuzzy Objective Function Algorithms". Norwell, MA, USA: Kluwer Academic Publishers, 1981.

[5] Grant, George, and Wilson, "Online topic modeling for real-time twitter search," 2011, TREC 2011 Notebook.

[6] Hong and Davison, "Empirical study of topic modeling in twitter," in Proc. of SOMA, ser. SOMA '10. NY, USA: ACM, pp. 80-88., 2010

[7] Francis and Flynn, "Text Mining Handbook", casualty Actuarial Society E-Forum, Spring 2010.

[8] Rao, "Building emotional dictionary for sentiment analysis of online news". World Wide Web, 2014. Vol.17, no.4, pp. 723-742. , 2014

[9] Chen, and Hassan, "A survey on the use of topic models when mining software repositories. Empirical Software Engineering”, ol.21, no.5, : pp. 1843-1919. , 2016

[10] Daud, A., et al., Knowledge discovery through directed probabilistic topic models: a survey. Frontiers of computer science in China, Vol.4, no.2, pp. 280-301., 2010

[11] Liu, and Zhang, "A survey of opinion mining and sentiment analysis, in Mining text data”, Springer. pp. 415-463, vol.320, 2012.

[12] Debortoli, "Text mining for information systems researchers: an annotated topic modeling tutorial". CAIS, Vol.39: pp. 7. 2016

[13] Sun, X., "Exploring topic models in software engineering data analysis: A survey", 17th IEEE/ACIS International Conference on Software Engineering, Artificial Intelligence, Networking and Parallel/Distributed Computing (SNPD). IEEE., 2016
[14] Minka, and Lafferty, " Expectation-propagation for the generative aspect model. in Proceedings of the Eighteenth conference on Uncertainty in artificial intelligence". 2002.

[15] Griffiths, Steyvers," "Finding scientific topics", Proceedings of the National academy of Sciences, 2004. Vol.101, pp. 5228-5235.

[16] Blei, $\mathrm{Ng}$, and Jordan, "Latent dirichlet allocation. Journal of machine Learning research",vol.3, pp.993-1022

[17] Mazarura, and Waal, "A comparison of the performance of latent Dirichlet allocation and the Dirichlet multinomial mixture model on short text", In Pattern Recognition Association of South Africa and Robotics and Mechatronics International Conference, pp. 1-6, IEEE., 2016. 